\title{
Prospects for Future Far-Infrared/Submillimeter Studies of the High-Redshift Universe
}

\author{
Andrew W. Blain \\ Institute of Astronomy, Madingley Road, Cambridge, CB3 OHA, UK
}

\begin{abstract}
Observations made using $C O B E$, SCUBA, ISO and MAMBO have provided a reasonable working knowledge of both the intensity of the submm and far-infrared background radiation and the source counts of luminous high-redshift dusty galaxies. However, because there are uncertainties in the background intensity determinations, the samples of detected galaxies are small, and most importantly, their redshift distributions are very incomplete, details of the evolution of dusty galaxies remain unresolved. The next steps forward in the field will be the launches of $S I R T F$ and $A S T R O-F$, the commissioning of SOFIA and new, more capable ground-based $\mathrm{mm} / \mathrm{submm}$-wave cameras - BOLOCAM, SHARC-II and SCUBA-II - the use of ultra-long duration balloon experiments, such as BLAST, the construction of ALMA and the arrival of FIRST, and ultimately the advent of space-borne far-infrared interferometers, such as $S P E C S$. There are also exciting prospects for direct $\mathrm{mm} / \mathrm{submm}$-wave CO-line redshift surveys using wide-band spectrographs. Using these new facilities, the number of high-redshift dusty galaxies known will be increased dramatically. Spectroscopy using SIRTF, SOFIA and FIRST will probe the astrophysical processes within these sources in detail, hopefully addressing the open question of the fraction of the counts and background radiation that is generated by the formation of high-mass stars and by active galactic nuclei (AGNs). The spatial and spectral structure of distant dusty galaxies will finally be resolved in detail using ALMA and SPECS.
\end{abstract}

\section{Introduction}

The determination of the intensity of extragalactic background radiation in the submm and far-infrared wavebands has been a very important development in observational cosmology (Puget et al. 1996; Hauser et al. 1998; Schlegel, Finkbeiner \& Davis 1998; Finkbeiner, Davis, \& Schlegel 2000). Over the same period, the first observations that were sufficiently deep to detect high-redshift galaxies in the $\mathrm{mm}$, submm and far-infrared wavebands were made using the 1.25-mm MAMBO bolometer camera at the IRAM 30-m telescope (Bertoldi et al. 2000), the $450 / 850-\mu \mathrm{m}$ SCUBA camera at the JCMT (Smail, Ivison, \& Blain 1997; for a current summary see Blain et al. 2000a) and the PHOT instrument aboard ISO (Kawara et al. 1998; Puget et al. 1999; Juvela, Mattila, \& Lemke 2000). Most of the results for both counts and background have been summarized elsewhere, see for example Figs. 9 and 12 in Blain et al. (1999b). 
Progress has been rapid: not only has the background radiation spectrum been determined for the first time, but most of the $850-\mu \mathrm{m}$ background radiation, and about $30 \%$ and $10 \%$ of the background at wavelengths of 450 and $175 \mu \mathrm{m}$ respectively has been accounted for as coming from individual detected galaxies.

This progress has been possible only because of tremendous improvements in the sensitivity and field of view of instruments in these wavebands. Many instrumental references can be found in Blain (1999a): to save space in this brief article they are generally not repeated here. The important pieces of missing information and the prospects for future developments of observations in the $\mathrm{mm}$, submm and far-infrared wavebands are discussed below.

\section{Key missing information - redshifts of submm-selected galaxies}

The $\mathrm{mm}$-far-infrared background radiation spectrum is generated by thermal emission from interstellar dust in all the galaxies that lie along the line of sight, right back to the epochs when the first dust was formed during the early process of galaxy formation. It potentially includes emission from redshifts beyond recombination, as a neutral Universe is transparent to long-wavelength radiation. The intensity and spectral shape of the background radiation provides information about the volume emissivity, intrinsic spectral energy distribution and redshift of dusty galaxies; however, this information is not unambiguous. The counts of galaxies that make up the brighter fraction of the population contributing to the background can be used to refine and confirm the results of analyses of the background spectrum, but unfortunately neither the values of these quantities nor different scenarios of galaxy evolution can be distinguished strongly. Indeed, from the background and count data alone, the median redshift of galaxies detected in deep SCUBA images at $850-\mu \mathrm{m}$ could plausibly lie between 1 and 5 . In order to discriminate between different well fitting models, measurements of redshift distributions are necessary; determinations of accurate counts at other $\mathrm{mm} / \mathrm{submm}$ wavebands are useful, but less important. For more details see Blain et al. (1999a,b)

Unfortunately, the redshift distribution of SCUBA galaxies is hard to determine for two reasons. First, it is difficult to identify counterparts for submmselected galaxies in other wavebands because their positions are relatively uncertain. The JCMT beam is 15 arcsec wide, and so the centroids of even the most significant detections are uncertain to within several arcseconds. Secondly, counterparts are reasonably expected to be extremely faint, perhaps so faint that optical spectroscopy will remain challenging until $N G S T$ is available (Smail et al. 2000).

Deep radio observations are very useful for finding the positions of counterparts (Ivison et al. 1998; 2000). The flux density ratio between the submm and radio wavebands can also be used to provide an indication of redshift (Carilli \& Yun 1999, 2000; Smail et al. 2000; Barger, Cowie, \& Richards 2000). Note however that the result is offset to lower values by the presence of any radio emission from a buried AGN, and depends on the dust temperature in the galaxy (Blain 1999b), with hotter galaxies at higher redshifts being difficult to distinguish from cooler ones closer by. Observations of high-resolution dust emission using mm-wave interferometer arrays can also be used to obtain better 
positions (Downes et al. 1999; Frayer et al. 2000; Gear et al. 2000); however, accurate relative optical-mm astrometry may be difficult in the absence of deep radio data because of the small fields of both the optical and mm-array images.

To confirm an identification beyond reasonable doubt, it is necessary to obtain an optical/near-infrared redshift for the candidate and then detect $\mathrm{CO}$ line emission at the same redshift and position. However, this is a slow process and an accurate redshift is essential. So far only three submm-selected galaxies have been observed in this way (Frayer et al. 1998, 1999; Kneib et al. in prep), less than $10 \%$ of those described in the literature. Future wide-band $\mathrm{mm}$ and submm spectrographs will improve this situation.

\section{Future progress}

At present, the SCUBA camera detects sources at the rate of about one per 8-hour shift at the JCMT. Within the next few years, new large-format $\mathrm{mm}$ submm cameras on ground-based telescopes - including BOLOCAM for the CSO/50-m LMT, SHARC-II (Dowell, Moseley, \& Phillips 2000) for the CSO and SCUBA-II for the JCMT - will offer more rapid detection rates and access new wavebands. At shorter wavelengths, the MIPS instrument onboard SIRTF, the HAWC camera aboard SOFIA and balloon-borne instruments such as BLAST (Devlin et al. 2000) will provide similar improvements over existing surveys. The simple increase in the detection rate of galaxies will assist followup studies by providing larger samples of objects to sift. In addition, the higher spatial resolution of images - from SCUBA-II at $450 \mu \mathrm{m}$, SHARC-II at $350 \mu \mathrm{m}$, and BOLOCAM at $1.1 \mathrm{~mm}$ on the LMT - will make the identification process easier. Wider survey fields and greater numbers of detections will allow largescale statistical studies of the distribution and clustering of high-redshift dusty galaxies to be carried out (Haiman \& Knox 2000).

There are also excellent prospects for more rapid detection of redshifted $\mathrm{CO}$ emission from candidate galaxies, mainly by increasing the bandwidth of instruments from the current maximum of $4 \mathrm{GHz}$ to several 10 's of $\mathrm{GHz}$. This will be sufficient to yield a realistic chance of detecting simultaneously adjacent CO lines - separated by $115 /(1+z) \mathrm{GHz}$ in the observer's frame for a galaxy at redshift $z$ - from a submm-selected galaxy, and so determine a redshift without requiring optical observations (see Blain et al. 2000b; Blain 2000).

In the longer term, the ground-based ALMA interferometer array and spaceborne mid- and far-IR interferometers such as $S P E C S$ will provide wide spectral coverage, sub-arcsec spatial resolution and great sensitivity. Using these instruments the detailed astrophysics within distant dusty galaxies will be resolved in detail. However, interferometers have small fields of view. Wide-field surveys made using the FIRST and Planck Surveyor space missions will be required to provide extensive lists of targets, in addition to the legacy of observations left by surveys made using SIRTF and ASTRO-F (Takeuchi et al. 1999).

\section{Summary}

A wide range of new instruments for studying the evolution of galaxies in the $\mathrm{mm}$, submm and far-infrared are proposed and under construction. Observa- 
tions using these facilities will enhance significantly the size and spatial resolution of catalogs of galaxies selected in these wavebands, and the amount of spectroscopic information available. The goal of understanding the history of dust-enshrouded galaxy formation and evolution in detail by determining the redshift distributions of galaxies selected in these wavebands will be realized.

Acknowledgments. I thank the Raymond \& Beverly Sackler Foundation, and ESO for support at the IAU meeting.

\section{References}

Barger, A. J., Cowie L. L., \& Richards, E. A., 2000, AJ, 119, 2092

Bertoldi, F., et al. 2000, A\&A, 360, 92

Blain, A. W. 1999a, ASP Conf. Ser. Vol. 191, 255 (astro-ph/9906141)

Blain, A. W. 1999b, MNRAS, 309, 955

Blain, A. W., Smail I., Ivison R. J., \& Kneib J.-P. 1999a, MNRAS, 302, 632

Blain, A. W., et al. 1999b, MNRAS, 309, 715

Blain, A. W. 2000, ASP Conf. Ser., in press (astro-ph/9911449)

Blain, A. W., et al. 2000a, ASP Conf. Ser. Vol. 193, 246 (astro-ph/9908111)

Blain, A. W., Frayer, D. T., Bock, J., \& Scoville, N. 2000b, MNRAS, 313, 559

Carilli, C. L., \& Yun, M. S. 1999, ApJ, 513, L13

Carilli, C. L., \& Yun, M. S. 2000, ApJ, 530, 618

Devlin, M., et al. 2000, Proc. UMass conference, in press

Dowell, C. D., Moseley, S. H., \& Phillips, T. G. 2000, ASP Conf. Ser., in press

Downes, D., et al. 1999, A\&A, 347, 809

Finkbeiner, D. P., Davis, M., \& Schlegel, D. J. 2000, ApJ, 544, 81

Frayer, D. T., et al. 1998, ApJ, 506, L7

Frayer, D. T., et al. 1999, ApJ, 514, L13

Frayer, D. T., et al. 2000, AJ, in press (astro-ph/0005239)

Gear, W. K., et al. 2000, MNRAS, in press (astro-ph/0007054)

Haiman, Z., \& Knox, L. 2000, ApJ, 530, 124

Hauser, M. G., et al. 1998, ApJ, 508, 106

Ivison, R. J., et al. 1998, MNRAS, 298, 583

Ivison, R. J., et al. 2000, MNRAS, 315, 209

Juvela, M., Mattila, K., \& Lemke, D. 2000, A\&A, in press (astro-ph/0005564)

Kawara, K., et al. 1998, A\&A, 336, L9

Puget, J.-L., et al. 1996, A\&A, 308, L5

Puget, J.-L., et al. 1999, A\&A, 345, 29

Schlegel, D. J., Finkbeiner, D. P., \& Davis, M. 1998, ApJ, 500, 525

Smail, I., Ivison, R. J., and Blain, A. W. 1997, ApJ, 490, L5

Smail, I., Ivison, R. J., Owen, F. N., et al. 2000, ApJ, 528, 612

Smail, I., et al. 2000, Proc. UMass conference (astro-ph/0008237)

Takeuchi, T. T., et al. 1999, PASP, 111, 288 